\title{
Variación estacional del tricograma en residentes chilenos
}

\author{
Mariana Kahn $\mathrm{Ch}^{1}$, Robinson G uerrero $\mathrm{A}^{2}$, \\ María Cristina Césped C ${ }^{3 a}$.
}

\section{Seasonal variation of trichogram in Chilean subjects}

Background: Human hair follicle experiments cyclical transformations throughout its life. These are rest (telogen phase), growth (anagen phase) and regression mediated by apoptosis (catagen). During summer the percentage of telogen increases and the percentage of anagen decreases. Aim: To determine the seasonal changes of scalp hair follicle phases among Chilean subjects. Material and methods: Retrospective analysis of 514 differential trichograms of normal scalps from subjects aged $36 \pm 14$ years, obtained between 1998 and 2008. Results: During summer and winter, the mean percentage of telogen was $20 \% \pm 10 \%$ and $16 \% \pm 10 \%$, respectively $(\mathrm{p}<0.01$ ). The figures for anagen in the same periods were $80 \% \pm 10 \%$ and $83 \% \pm 10 \%$, respectively. Conclusions: This study confirms the presence of seasonal variations in normal trichogram (Rev Méd Chile 2009; 137: 1437-40).

(Key w ords: Alopecia; Hair follicle; Scalp)

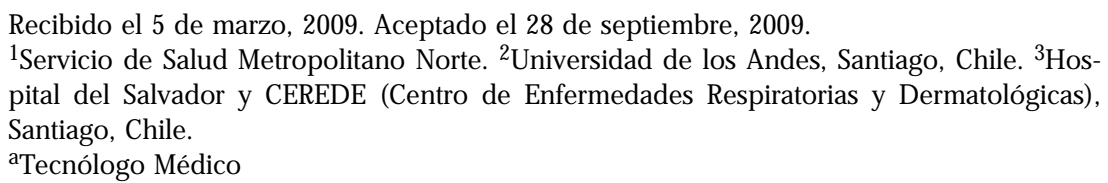

$\mathrm{D}$ esde los inicios de la humanidad el cabello ha tenido una importancia trascendental desde el punto de vista religioso, racial, sexual y ornamental. En los seres humanos el pelo es un vestigio evolutivo, a diferencia de otros seres vivos, donde cumple funciones de protección y regulación de la temperatura ${ }^{1}$.

El número de pelos que se desprenden normalmente es de aproximadamente 100 por día y esta tasa aumenta al final del verano y principios de otoño, quizás debido a efectos de la mayor radiación solar y temperatura ${ }^{2,3}$.

Correspondencia a: Dra. Mariana Kahn Chernilo. Alejandro Valdés Riesco 2624, Providencia, Santiago, Chile. Fono: 4150699. E mail: marianakahnchernilo@hotmail.com
Las variaciones de densidad varían según diferentes autores pero la media estimada es de 615 pelos $/ \mathrm{cm}^{2}$ en el tercer decenio de vida y 485 pelos/ $\mathrm{cm}^{2}$ en el quinto decenio ${ }^{4,5}$.

El folículo piloso en los seres humanos experimenta a lo largo de toda la vida transformaciones cíclicas: reposo (telógeno), crecimiento (anágeno) y regresión mediada por apoptosis (catágeno $)^{6}$. El folículo piloso es el único órgano en los mamíferos que sufre este tipo de cambios cíclicos. Estas transformaciones están controladas por cambios en factores locales de señalización los cuales a su vez se ven modulados por la expresión y actividad de citoquinas, hormonas y neurotransmisores. Se ha demostrado que existe un reloj que controla el ciclo folicular dentro del mismo folículo piloso; sin embargo, se desconocen los meca- 
nismos moleculares exactos que permiten la oscilación de este sistema ${ }^{7}$.

El tricograma es un estudio que consiste en extraer manualmente 50 a 100 pelos de distintas partes del cuero cabelludo. Los pelos luego son examinados mediante un microscopio para determinar qué porcentaje de éstos se encuentra en cada una de las tres fases del ciclo del folículo piloso: telógeno (Figura 1), anágeno (Figura 2) o catágeno. Los porcentajes normales son de $85 \%$ de pelos en anágeno y 15\% en telógeno. En general se utiliza el tricograma diferencial que consiste en dos muestras; una correspondiente a cuero cabelludo normal y otra correspondiente a una zona de menor densidad. Es posible seguir en el tiempo los distintos tipos de alopecía mediante la repetición de este examen. El análisis cuantitativo del cambio de tricograma es un buen método para evaluar formulaciones capilares y para definir el tratamiento y prevención de la caída del cabello ${ }^{8,9}$.

El máximo de cabellos en telógeno precede por uno a dos meses la máxima caída de cabello, lo que es compatible con una duración promedio de la etapa de telógeno de dos a tres meses. A partir de esto se justifica la elección del porcentaje de telógeno como criterio para evaluar la pérdida de cabello ${ }^{10}$.

El hecho de que los folículos pilosos del ser humano experimenten cambios cíclicos al igual que otros mamíferos y sean influenciados por hormonas y otras substancias hace surgir una pregunta de interés evolutivo, así como también de importancia práctica con respecto a la determi-

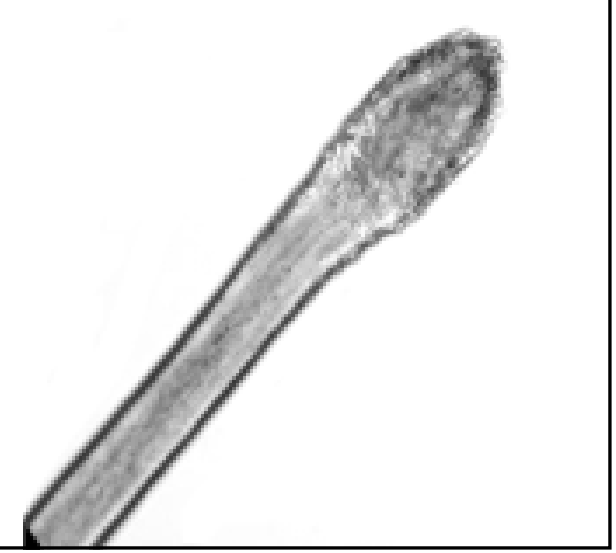

Figura 1. Folículo piloso en fase de telógeno. Preparación de microscopia óptica al fresco sin tinción (10x). nación de efectos farmacológicos: ¿varía estacionalmente el crecimiento del cabello humano?

Se ha visto que el porcentaje de telógeno aumenta a finales del verano y principios de otoño y que el porcentaje de anágeno disminuye en este mismo período ${ }^{2,3}$. Sin embargo, no existen estudios nacionales que analicen la variación estacional de las fases del ciclo del folículo piloso.

El objetivo de este estudio es determinar la variación estacional de las fases del ciclo del folículo piloso en el cuero cabelludo en pacientes residentes en Chile mediante el análisis cuantitativo con tricograma.

\section{MATERIAL Y MÉTODO}

La hipótesis de trabajo consistió en demostrar si existía variación estacional del porcentaje de telógeno y anágeno en el tricograma en pacientes residentes en Chile. Se planteó que en verano, aumenta el porcentaje de telógeno y disminuye el porcentaje de anágeno.

Consiste en un estudio observacional descriptivo y retrospectivo de análisis de los tricogramas diferenciales de cuero cabelludo realizados en pacientes estudiados en CEREDE (Centro de Enfermedades Respiratorias y Dermatológicas, Santiago de Chile) desde enero a diciembre de los años 1998 a 2008. Todos los tricogramas incluidos en el estudio correspondieron a exámenes con extracción manual de cabellos. Las variables estu-

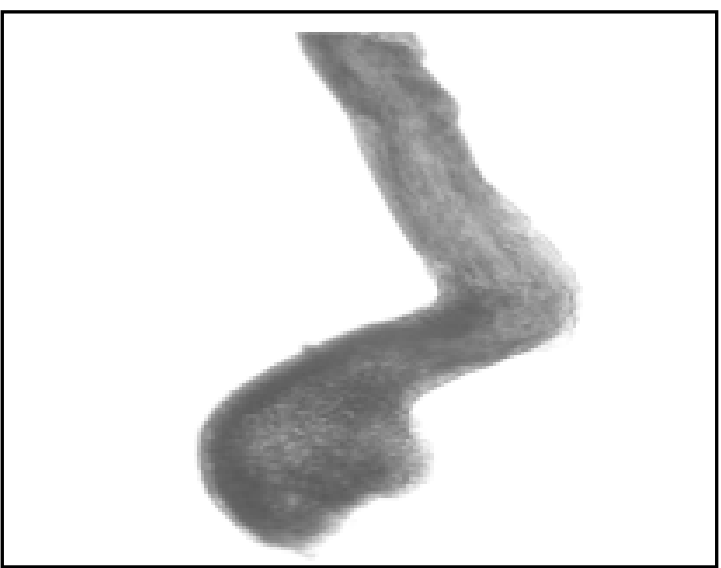

Figura 2. Folículo piloso en fase de anágeno. Preparación de microscopia óptica al fresco sin tinción (10x). 
diadas fueron edad, sexo, porcentaje de telógeno y porcentaje de anágeno. Se consideró como meses de verano en el hemisferio sur desde octubre hasta marzo (inclusive) y como meses de invierno desde abril a septiembre (inclusive).

Se utilizó sólo la información contenida en los tricogramas diferenciales con respecto al cuero cabelludo normal.

Para comprobar la distribución normal de los porcentajes de telógeno y anágeno se utilizó la prueba de Shapiro-Wilk. Para el análisis de las diferencias de telógeno y anágeno entre estaciones del año se utilizó la prueba de t de Student para diferencia de medias de dos muestras independientes. Para el análisis estadístico se utilizó el programa Stata IC 10.0. El nivel de significación utilizado fue de 0,05.

\section{Resultados}

Se analizaron 514 tricogramas entre enero y diciembre de los años 1998 a 2008. El promedio de edad fue de $35,5 \pm 13,5$ años. El 38,5\% de los tricogramas comesponden a pacientes de sexo masculino (198 tricogramas) y $61,5 \%$ de los tricogramas comesponden a pacientes de sexo femenino (316 tricogramas) (Tabla 1).

En la Figura 3 se observa el porcentaje de telógeno según mes del año. Se aprecia que en octubre existe un máximo de pelos en telógeno $(24,4 \%)$, valor que en noviembre disminuye a $18 \%$ para luego permanecer más o menos constante hasta marzo. A partir del mes de abril el porcentaje de telógeno disminuye hasta llegar a un mínimo de $14 \%$ en junio. A partir de julio el porcentaje de telógeno aumenta nuevamente hasta llegar al máximo previamente descrito.
En la Figura 4 se observa el porcentaje de anágeno según mes del año. Se aprecia que en el mes de octubre existe un mínimo de pelos en anágeno (75,9\%). Entre noviembre y enero el porcentaje de anágeno se mantiene más o menos constante en $81 \%$, para luego caer a 78,9\% en el mes de febrero. A partir de marzo este valor comienza a aumentar hasta llegar a un máximo de 85,7\% en el mes de junio. A partir de julio el porcentaje de anágeno disminuye nuevamente hasta llegar al mínimo previamente descrito.

El promedio del porcentaje de telógeno fue de $19,7 \%$ en verano y de $16,4 \%$ en invierno y el promedio del porcentaje de anágeno fue de 79,9\% en verano y de $83,0 \%$ en invierno.

$\mathrm{Al}$ realizar el análisis estadístico se vio que los porcentajes de telógeno y anágeno distribuían normal tanto en verano como en invierno. La diferencia del porcentaje de telógeno entre verano e invierno fue significativa ( $p=0,0002$ ), igual que la diferencia del porcentaje de anágeno entre verano e invierno ( $p=0,0005)$.

Lo anterior demuestra que sí existe variación estacional de las fases del ciclo del folículo piloso en el cuero cabelludo normal en residentes chilenos con diferencia estadísticamente significativa con mayor porcentaje de telógeno en verano y mayor porcentaje de anágeno en invierno.

\section{Discusión}

Los resultados del presente estudio coinciden con otros estudios internacionales con respecto a la estacionalidad de las fases del ciclo del folículo piloso en el cuero cabelludo2,3. Esta variación

Tabla 1. C aracterísticas demográficas y porcentajes de telógeno y anágeno según estación del año. Los resultados se presentan como promedio \pm DS

\begin{tabular}{|lc|}
\hline Variable & Valor \\
\hline Edad (años) & $35,5 \pm 13,5(7-79)$ \\
Sexo masculino & $198 / 514(38,5 \%)$ \\
Sexo femenino & $316 / 514(61,5 \%)$ \\
Telógeno verano (\%) & $19,7 \pm 10,3$ \\
Anágeno verano (\%) & $79,9 \pm 10,3$ \\
Telógeno invierno (\%) & $16,4 \pm 10,2$ \\
Anágeno invierno (\%) & $83,0 \pm 10,4$ \\
\hline
\end{tabular}


estacional podría explicarse por la temperatura, luminosidad, radiación solar, factores hormonales y otros. No están claros todavía los mecanismos exactos que permitirían la oscilación armónica de este sistema ${ }^{7}$.

Se ha visto además que pelos de otras partes del cuerpo como barba y muslos tendrían también variación estacional en las fases del ciclo del folículo piloso ${ }^{2}$. Es imposible saber si los ritmos circadianos anuales se relacionan con la historia evolutiva del ser humano o si tienen un significado adaptativo. La sobrevivencia del pelo en el cuero cabelludo a diferencia del existente en el resto del cuerpo se presume serviría como protección contra los rayos solares del mediodía para un animal bípedo.

La mantención de bajos niveles de telógeno durante el invierno y por ende la menor caída de cabello durante este período podría corresponder a una ventaja selectiva. De esta manera, se pospondría la caída del cabello hasta finales del verano y principios de otoño lo que le permitiría al hombre estar protegido de los rayos solares durante la época de mayor radiación. De hecho, muchos individuos notan un aumento de la caída del cabello en ciertos períodos del año, generalmente en otoño (como consecuencia del aumento del porcentaje de telógeno a fines del verano) y algunas veces en primavera. En CEREDE se ha visto también un aumento de la consulta dermatológica por efluvio telógeno entre los meses de noviembre y marzo que coincide con los hallazgos de este estudio.

Este estudio, el primero a nivel nacional en su tipo, muestra las variaciones naturales de las fases del ciclo del folículo piloso. Es muy importante considerar estas fluctuaciones al momento de

\section{REFERENCIAS}

1. Whiting D. Chronic telogen effluvium. Dermatologic Clinics 1996; 14: 723- 31.

2. Randall V, Ebling FJG. Seasonal changes in human hair growth. Br J Dermatol 1991; 24: 145-51.

3. Courtois M, Loussouarn G, Hourseau S, Grower JF. Periodicity in the growth and shedding of hair. $\mathrm{Br} \mathrm{J}$ Dermatol 1996; 134: 47-54.

4. Giacometti L. The anatomy of the human scalp. Adv Biol Skin 1965; 6: 97-120.

5. Самacho F, Montagna W. Algunos aspectos de la fisiología del folículo piloso. En: Camacho F, Mon-

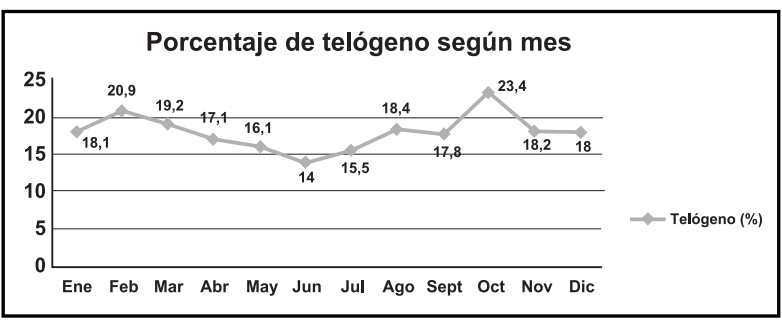

Figura 3. Porcentaje de pelos en telógeno según mes del año.

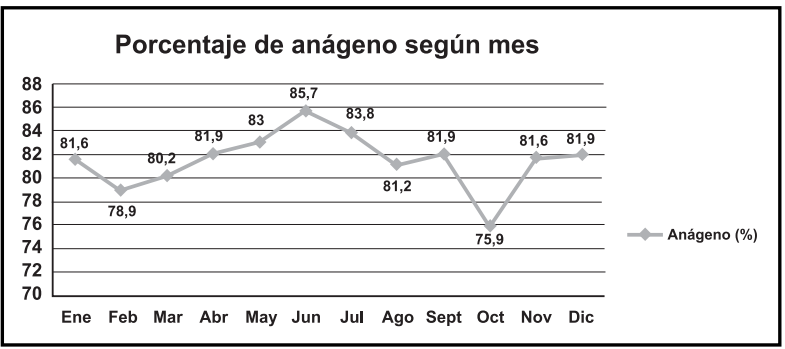

Figura 4. Porcentaje de pelos en anágeno según mes del año.

evaluar formulaciones capilares y en la definición del tratamiento y prevención de la caída del cabello. Sería interesante diseñar un estudio prospectivo donde se midiera el porcentaje de telógeno y anágeno en el cuero cabelludo en distintas estaciones del año durante varios años juntos con otras variables como temperatura, luminosidad, radiación solar, niveles hormonales y otros para así determinar cuáles son los factores involucrados en esta variación estacional.

\section{Agradecimientos}

Se agradece a Óscar Figueroa Castro, tecnólogo médico de la Fundación Médica San Cristóbal, Santiago, Chile, por su contribución con las imágenes de microscopia.

tagna W. Tricología. Enfermedades del folículo pilosebáceo. Grupo Aula Médica S.A. 1996, Madrid, España, capítulo 21, pgs. 36-9.

6. PAus R. Principles of hair cycle control. J Dermatol 1998; 25: 793-802.

7. Krause K, FoitziK K. Biology of the hair follicle: the basics. Semin Cutan Med Surg 2006; 25: 2-10.

8. Chamberlain AJ, Dawber RP. Methods of evaluating hair growth. Australas J Dermatol 2003; 44: 10-8.

9. Barman JM, Astore I, Pecoraro V. The normal trichogram of the adult. J Invest Dermatol 1965; 44: 233-6.

10. Courtois M, Loussouarn G, Hourseau C, Grower JF. Hair cycle and alopecia. Skin Pharmacol 1994; 7: 84-9. 